\title{
Pneumonitis complicating selective intra-arterial chemotherapy for locally advanced breast carcinoma
}

\author{
Andrew D McLean, Julie C Doughty, Allan W Reid, Douglas H McCarter, Elizabeth \\ Kane, Colin S McArdle
}

\author{
Summary \\ We describe a case of pneumonitis in a \\ patient receiving selective intra-arterial \\ chemotherapy for locally advanced \\ breast cancer.
}

Keywords: breast cancer, pneumonitis, chemotherapy

Despite the introduction of breast screening, $15 \%$ of patients with breast cancer still present survival of patients treated by local therapy alone is only $20 \%$, and in an attempt to address this problem systemic treatment is now routinely offered to these patients.

Studies show that although overall survival is not significantly increased by the addition of chemotherapy, the time to first progression is significantly delayed by systemic therapy. This is due almost entirely to the time to locoregional progression rather than time to distant metastases.

Since the activity of most drugs is dosedependent, regional chemotherapy which aims to deliver a high dose of drug directly to the tumour-bearing organ may be even more effective for this group of patients. As well as increasing the local concentration of drug, theoretically less drug will enter the systemic circulation and there will be less systemic toxicity. ${ }^{2}$ Nevertheless, depending upon how much drug is extracted or metabolised during first pass through the target organ, systemic toxicity may still occur.

Glasgow Royal

Infirmary, Glasgow

G31 2ER, UK

University

Department of

Surgery,

AD McLean

JC Doughty

E Kane

CS McArdle

Department of

Radiology

AW Reid

DH McCarter

Correspondence to $\mathrm{Mr}$ Andrew D McLean,

Department of General

Surgery, Law Hospital,

Carluke, Lanarkshire ML8

5ER, UK

Accepted 28 June 1995

\section{Case report}

A previously fit 40 -year-old woman presented with histologically proven locally advanced inflammatory breast carcinoma. Staging revealed no evidence of axillary lymph nodes or systemic disease. Using our previously described technique, ${ }^{3}$ platinum occlusion coils were angiographically placed in the distal internal mammary artery. Following this, regional intra-arterial chemotherapy with mitomycin $\left(7 \mathrm{mg} / \mathrm{m}^{2}\right)$, mitozantrone $\left(7 \mathrm{mg} / \mathrm{m}^{2}\right)$ and methotrexate $\left(30 \mathrm{mg} / \mathrm{m}^{2}\right)$ was administered over four hours via the internal mammary artery. Four doses were administered at monthly intervals. A complete clinical response of her breast carcinoma was observed. Systemic with locally advanced disease. The five-year

toxicity was minimal and her lungs appeared normal (figure 1). However, three days following her last dose of chemotherapy she developed rapidly worsening dyspnoea and cough necessitating hospital admission.

Her condition rapidly worsened over four days with progressive respiratory failure. Chest radiography and computed tomography (CT) showed bilateral hilar enlargement with increased interstitial markings throughout each lung and patchy alveolar infiltrates (figure 2). Bilateral pleural effusions were also present. Transbronchial lung biopsy showed only nonspecific inflammatory changes and bronchialveolar lavage results were non-specific with no evidence of active lung infection. A diagnosis of drug-related pneumonitis was made and the patient was commenced on high-dose intravenous methylprednisolone. She made a rapid and full recovery.

Two months later she underwent mastectomy and axillary dissection. There was no pathological evidence of residual carcinoma in the breast and a solitary deposit was found in the axillary fat. No metastases were found in the lymph nodes.

\section{Discussion}

Pneumonitis is a well-recognised side-effect of many drugs, including the cytotoxics methotrexate and mitomycin (box 1). Pleural and mediastinal reactions may also occur. The condition is relatively rare, unpredictable and usually of insidious onset. It may occur up to

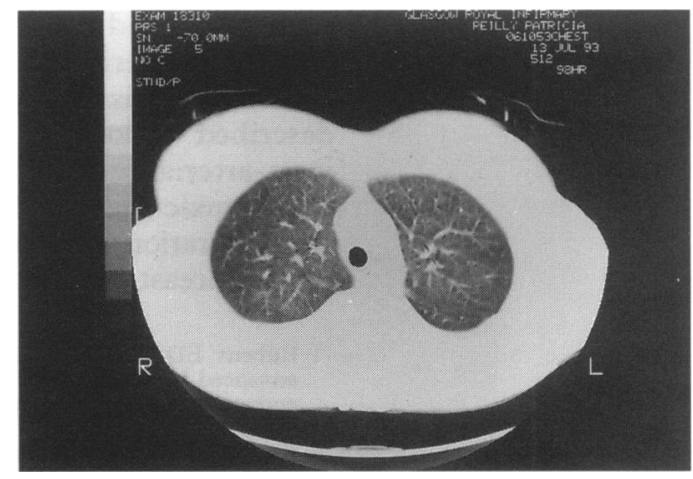

Figure 1 Initial CT scan of chest at the time of the fourth course of regional chemotherapy, showing normal lungs. 


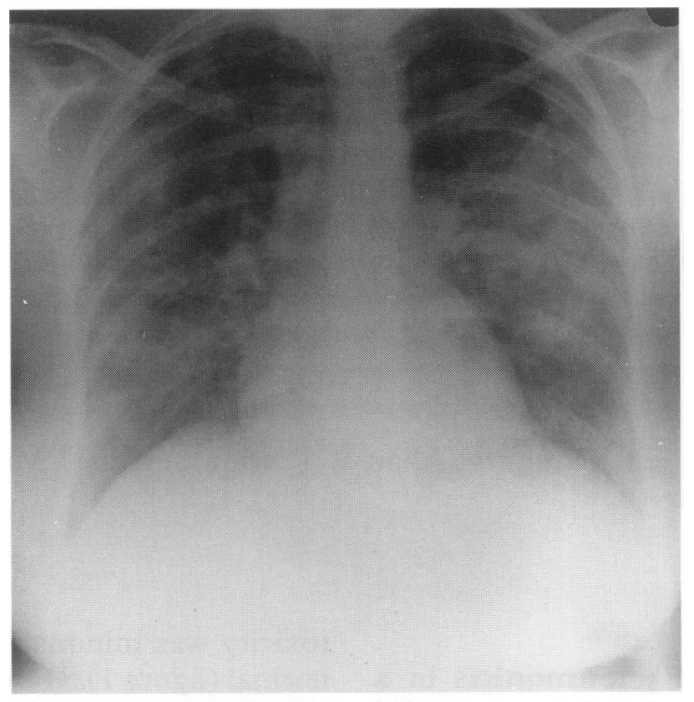

A

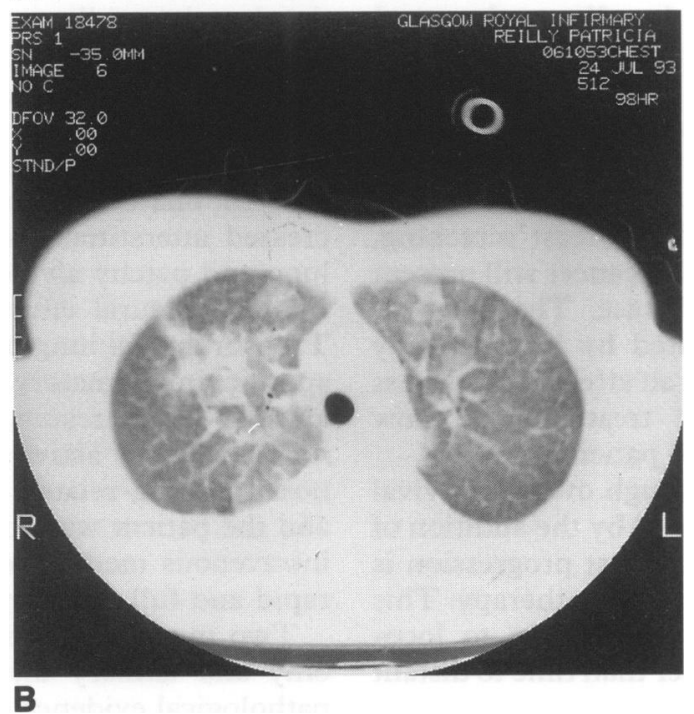

Figure 2 Chest radiograph (A) and CT scan (B) following the onset of respiratory symptoms showing ill defined and bilateral alveolar shadowing

four weeks after administration ${ }^{4}$ and is not dose-related. ${ }^{5}$ The diagnosis ultimately remains one of exclusion, particularly of infective causes and lymphangitis carcinomatosis, and although no prospective trial has occurred, up to $75 \%$ of cases show a good response to systemic corticosteroids.

The condition has been described after many routes of administration, both systemic and regional, including intrathecal, intraperitoneal and intra-uterine administration. To our knowledge this is the first time it has been described following administration via the intra-arterial route. Some systemic absorption of cytotoxic agents following internal thoracic administration of cytotoxics for locally advanced breast carcinoma is both inevitable and

1 Rubens ED, Bartelink H, Engelsman E, et al. Locally advanced breast cancer. The contribution of cytotoxic and endocrine treatment to radiotherapy. Eur $f$ Cancer Clin Oncol 1989; 25: 667-8.

2 Kerr DJ, Los G. Pharmacokinetic principles of locoregional chemotherapy. Cancer Surv 1993; 17: 105-22.

3 McCarter DH, Doughty J, Cooke TG, McArdle CS, Reid AW. Embolisation of the distal internal mammary artery as an adjunct to regional chemotherapy. $\mathcal{f}$ Vasc Intervent Radiol 1995; 6: 249-51.

\section{Drug causes of pneumonitis}

- Cytotoxics:

BLEOMYCIN

BUSULPHAN

METHOTREXATE

MITOMYCIN C

nitrosoureas

chlorambucil

melphalan

cyclophosphamide

cytosine arabinoside

6-mercaptopurine

azathioprine

- Anti-arrhythmics:

AMIODARONE

tocainamide

procainamide

quinidine

- Hypotensives:

HYDRALAZINE

methyl-DOPA

hexamethonium

- Anticonvulsants:

phenytoin

carbamazepine

- Anti-inflammatories:

SULPHASALAZINE

penicillamine

gold

- Antibiotics:

NITROFURANTOIN

sulphonamides

penicillins

PAS

- Others:

chlorpropamide

cromoglycate

pituitary snuff

This list is not comprehensive and includes drugs causing a wide range of pneumonitis/alveolitis syndromes. Those drugs recognised as most frequently causing pneumonitis are listed in capitals.

\section{Box 1}

\begin{tabular}{|l|}
\hline Summary/learning points \\
\hline - systematic toxicity, although decreased, still \\
occurs with regional intra-arterial \\
administration of cytotoxic drugs \\
- cytotoxic pneumonitis, although having \\
characteristic features, remains a diagnosis of \\
exclusion and bronchoscopy and biopsy are \\
useful in this role \\
- the onset of pneumonitis may be delayed up to \\
several weeks \\
- true drug-induced pneumonitis usually \\
responds well to corticosteroids
\end{tabular}

Box 2

desirable for control of systemic metastatic disease. The possibility of systemic sideeffects, especially those which are idiosyncratic and unrelated to dose, should always be borne in mind.

4 Elasser $S$, Dalquen $P$, Soler $M$, Perruchoud AP. Methotrexate-induced pneumonitis: appearance four weeks after discontinuation of treatment. Am Rev Respir Dis 1989; 140: 1089-92.

5 Searles G, McKendry RJ. Methotrexate pneumonitis in rheumatoid arthritis: potential risk factors. Four case reviews and a review of the literature. $\mathcal{F}$ Rheumatol 1987; 14: 1164-71. 